\title{
ICRF-Code Benchmark Activity in the Framework of the European Task-Force on Integrated Tokamak Modelling
}

\author{
R. Bilato*, D. Coster*, R. Dumont ${ }^{\dagger}$, T. Johnson**, H.-J. Klingshirn*, E. Lerche ${ }^{\ddagger}$, \\ O. Sauter ${ }^{\S}$, M. Brambilla*, L. Figini ${ }^{\mathbb{T l}}$, D. Van Eester ${ }^{\ddagger}$, L. Villard ${ }^{\S}$, D. Farina ${ }^{\mathbb{q}}$ and \\ ITM-TF contributors"
}

\author{
* MPI for Plasma Physics, EURATOM Association, Boltzmannstr. 2, 85748 Garching, Germany \\ $\dagger$ CEA, IRFM, F-13108 Saint-Paul-lez-Durance, France \\ ** School of Electric Engineering, KTH, Association VR-Euratom, Sweden \\ * Association EURATOM-Belgian State, LPP-ERM-KMS, TEC-partner, Brussels, Belgium \\ $\S$ CRPP, Association EURATOM-Confèdèration Suisse, EPFL, Lausanne, Switzerland \\ II Istituto di Fisica del Plasma CNR, EURATOM-ENEA-CNR Association, Milano, Italy \\ "See the Appendix to the paper of G. Falchetto et al., Nucl. Fus. submitted.
}

\begin{abstract}
The grand aim of the Integrated Tokamak Modelling (ITM) task-force is to provide a flexible, modular and reliable plasma simulator in view of planning and analyzing ITER discharges. Since radio-frequency (rf) heating in the ion cyclotron range of frequencies (ICRF) is foreseen as one of the main additional heating systems in ITER, physics modules that simulate ICRF wave propagation and absorption are necessary for the ITM project.

Here, we report on the status of the benchmark activity of ICRF codes, already imported in ITM environment platform. We consider various scenarios for ITER, limiting the comparisons to wave propagation and absorption in Maxwellian plasmas.
\end{abstract}

Keywords: ICRF, full-wave solver, ITM

PACS: $52.40 . \mathrm{Db}, 52.50 . \mathrm{Qt}, 52.55 . \mathrm{Fa}$

\section{INTRODUCTION}

As for all the physics modules imported on the ITM platform [1], also the ICRF codes have to pass an ongoing process of verification, i.e. testing whether the implementation accurately represents the physical model claimed by the developers, and validation, i.e. establishing how accurately the implemented model represents the experiments. Verification can be performed in a restricted range of parameters for which analytical solutions are known, or in a more extended and realistic parameter domain by comparing the results of different codes which aim to describe the same physical phenomena. However, having more than one module describing the same physical phenomena is not just desirable for code verification, but it adds robustness and flexibility to the whole ITM project if the modules are easily interchangeable in the plasma simulator, which is the final goal. Therefore, most of the initial efforts have been to standardize the data to be exchanged and in building the necessary software infrastructure for the data storage and transfer. Although still in progress, this initial process has produced the first versions of Consistent Physical Objects (CPO) [2] and of Universal Access Layer (UAL) [3] infrastructure, and made operative the project. It is important to note that the definition of CPOs has required a rethinking of conventions. In particular, a coordinate systems classification has been compiled [4].Therefore, already in its developing phase, the ITM environment offers an unique platform for code benchmarking since most of the distresses typical of the benchmarking activity are avoided, such as difficulties mainly related to input/output formats, data transfer, and conventions [5].

Before discussing the results of the benchmark involving ICRF full-wave codes presently imported on the ITM platform, we present a few features of the ITM platform which have been particular useful for our activity.

\section{INTEGRATION: CPO AND UAL}

The integration concept in ITM project is three-fold [1]. Firstly, it is necessary to build a platform for code integration of physics modules that already exist and of those that will be written in the future. Secondly, it is essential to guarantee the discipline integration: the final goal of every modelling is to interpret and predict experimental data. Therefore, a direct comparison with experiments is possible if the engineering parameters are used as much directly as possible in the modelling, and if the results of the simulations are stored as quantities that can be compared with measurements. 
TABLE 1. ICRF full-wave codes presently imported on the ITM linux cluster.

\begin{tabular}{l|c|c|c|c|c|c}
\hline Code & Version & Geometry & Approx. & Harmonics & MC & Num. Method \\
\hline EVE [6] & 1.9 .10 & toroidal, axisymmetric & FLR & $0,1,2$ & yes & $\begin{array}{c}\text { toroidally and poloidally spectral } \\
\text { radially FEM }\end{array}$ \\
\hline CYRANO [7] & 4.1 & toroidal, axisymmetric & FLR & $0,1,2$ & no & $\begin{array}{c}\text { toroidally and poloidally spectral } \\
\text { radially FEM }\end{array}$ \\
\hline LION [8] & 1.5 & toroidal, axisymmetric & $\begin{array}{c}\text { FLR } \\
\text { (fast) }\end{array}$ & $0,1, . ., 10$ & no & $\begin{array}{c}\text { toroidally spectral } \\
\text { radially and poloidally FEM }\end{array}$ \\
\hline TORIC [9] & 6.1 & toroidal, axisymmetric & FLR & $0,1,2$ & yes & $\begin{array}{c}\text { toroidally and poloidally spectral } \\
\text { radially FEM }\end{array}$ \\
\hline
\end{tabular}

Thirdly, it is crucial to support physics integration: in view of achieving a high level of granularity to allow flexibility in building workflow, physics interfaces between codes are necessary. For instance, many of the ICRF codes are already interfaced with Fokker-Planck (FP) solvers, as needed to perform selfconsistent simulations and eventually to provide sources to transport codes. However, the interfaces are often hardwired in the codes, and the replacement of one of the modules requires to re-write parts of the interfaces. Thus, one of the key goals of ITM is to provide physics modules that fill the gaps between codes that address different but connected physical phenomena. Staying with the example of ICRF and FP codes, a standalone interface is necessary that builds the quasilinear operator from the wave fields evaluated by ICRF codes. In addition, physics integration is necessary to bridge between modelling results and diagnostics measurements. This can be achieved with synthetic diagnostics that translate modelling results in directly measurable quantities, e.g. those of fast ion detectors, of neutron particle analyzers, of phase-contrast-imaging systems, and of neutron rate detectors, just to cite a few examples close to ICRF field. These interfaces are crucial also for the validation procedure of ICRF and Fokker-Planck codes.

The way envisaged by ITM to achieve integration is to transfer the interface functionality directly to the structure of the data exchanged among the physics modules. In short, the data are structured according to physical and engineering concepts. Just to cite the CPOs used in our benchmark, all the information concerning the equilibrium are collected and documented in the equilibrium CPO; all the information concerning the plasma profiles and plasma composition are in the coreprof $\mathrm{CPO}$; the antenna geometry is defined in the antennas $\mathrm{CPO}$; and finally the waves $\mathrm{CPO}$ contains the wave information necessary as input of an ICRF code, such as the toroidal wavenumbers, frequencies, and the amount of power coupled to each toroidal mode. In turn, the output of full-wave codes is stored in the fields of the same waves CPO. Many fields and structured fields of the CPOs are dedicated to document the CPOs.

The functionality of CPOs is guaranteed by the UAL, developed by the ITM: this library makes the reading/writing of the CPOs transparent to the languages mostly used in our community, namely Fortran, C, python, and Matlab.

\section{BENCHMARK RESULTS}

Presently there are four ICRF full-wave codes adapted to deal with CPOs in input and in output. Their main characteristics are summarized in table 1 . For the benchmark two preliminary shotfiles have been built corresponding to full-field (activated) and half-field (preactivated) phases of ITER [5]. The main parameters are summarized in table 2. The equilibrium CPOs were filled with CHEASE code [10] for both scenarios. In the antennas CPO the straps have been idealized as straight conductors of length $120 \mathrm{~cm}$, located at $8.45 \mathrm{~m}$ from the geometrical center of the torus, and carrying constant currents. The poloidal cross sections of the magnetic surfaces with the position of the main ICRF resonances are shown in figures (1.a-2.a). Here, we consider only propagation and absorption in Maxwellian plasmas. In the full-field case, $\mathrm{He}^{3}$ concentration varies from $0 \%$ to $10 \%$, and correspondingly D concentration decreases from $52 \%$ to $32 \%$. For the half field case, the $\mathrm{H}$ concentration changes from $0 \%$ to $19 \%$. In both cases, by increasing the minority concentration the ion-ion resonance moves towards the high field side (the left hand side in figures (1.a-2.a)). The CPOs are indexed with shot and run numbers, and, as for the experimental shotfiles, there is an index representing time. Here each time slice is characterized by a specific plasma composition, frequency, and $n_{\varphi}$, whereas all the other parameters are fixed. In reference to the numerical resolution, the simulations with EVE have been done using 480 radial points in plasma, and 129 poloidal modes; CYRANO runs have been performed with 500 radial points and 96 poloidal modes; the results of LION have been obtained with 256 radial points and 200 poloidal angles; TORIC has been run with 500 radial points and 127 poloidal modes. In these ICRF scenarios for ITER, the fundamental IC resonance of the minority species coincides with the second IC harmonics of one of the majority species. This is 

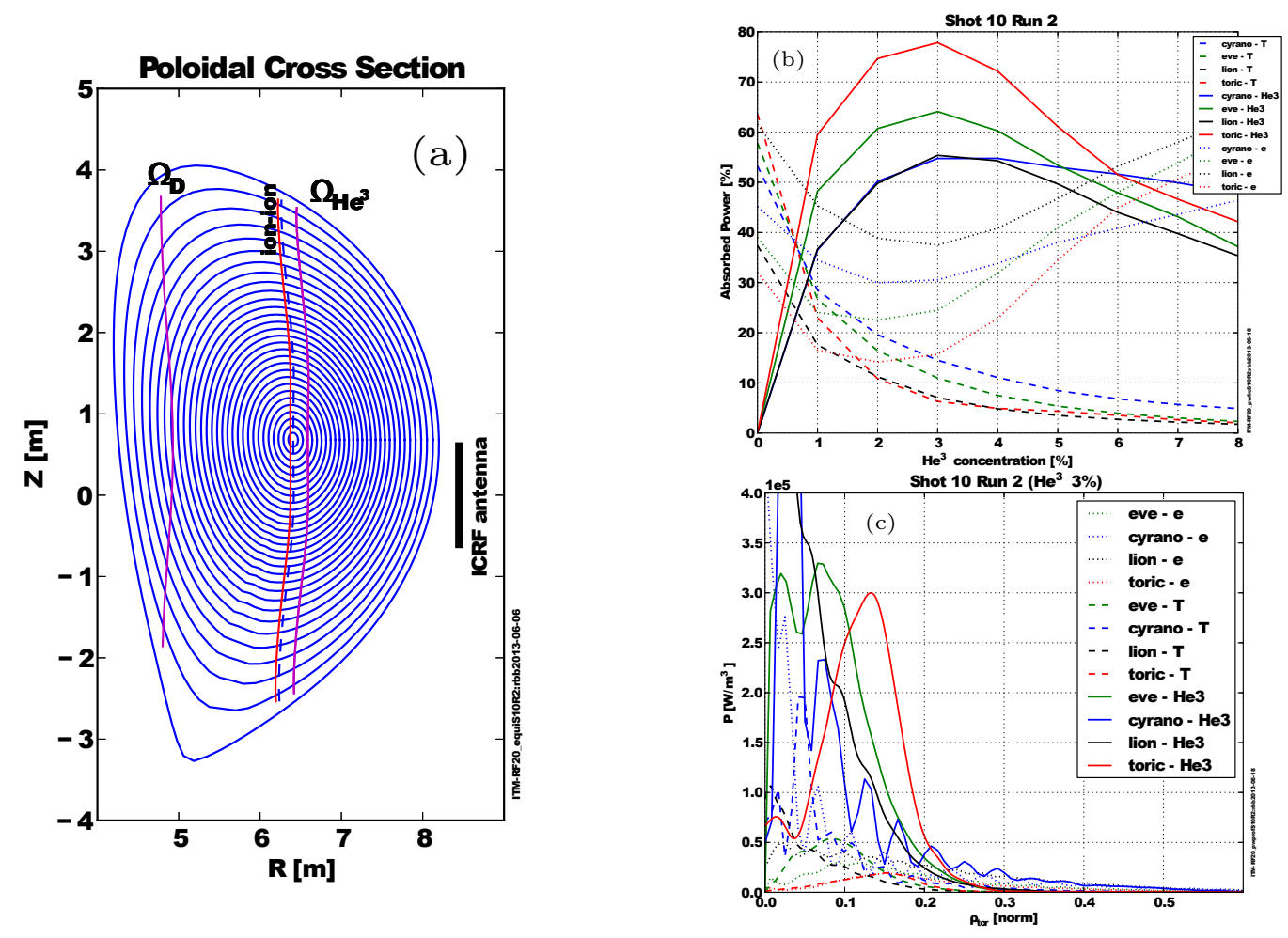

FIGURE 1. Full-field ITER case: (a) Poloidal cross section of the magnetic configuration; (b) Power repartition as function of $\mathrm{He}^{3}$ concentration; (3) Absorbed-power profiles for $3 \%$ of $\mathrm{He}^{3}$. In these simulations $\mathrm{f}=52.5 \mathrm{MHz}$ and $n_{\varphi}=27$.

TABLE 2. Main parameters of the full- and half-field ITER scenarios used for the benchmark. The concentrations of D and He ${ }^{3}$ for the full-field case, and of $\mathrm{He}^{4}$ and $\mathrm{H}$ for the half-field scenario are varied in such a way to guarantee the charge neutrality. The assumed coupled rf power is $10 \mathrm{MW}$. The Shot and Run are the numbers used to catalogue the shotfile.

\begin{tabular}{l|c|c|c|c|c|c|c|c|c}
\hline Case & Shot/Run & $\begin{array}{c}\mathrm{I}_{p} \\
(\mathrm{MA})\end{array}$ & $\begin{array}{c}\mathrm{B}_{0} \\
(\mathrm{~T})\end{array}$ & $\begin{array}{c}\mathrm{T}_{e} \\
(\mathrm{keV})\end{array}$ & $\begin{array}{c}\mathrm{T}_{i} \\
(\mathrm{keV})\end{array}$ & $\begin{array}{c}\mathrm{n}_{e} \\
\left(10^{19} \mathrm{~m}^{-3}\right)\end{array}$ & $\begin{array}{c}\mathrm{f} \\
(\mathrm{MHz})\end{array}$ & $\mathrm{n}_{\varphi}$ & Ion Species \\
\hline full-field & $10 / 2$ & 15. & 5.3 & 24.8 & 21.0 & 10.3 & $52.5,50.5$ & $\pm(27,50)$ & $\mathrm{D}\left(\mathrm{He}^{3}\right), \mathrm{T}(46 \%), \mathrm{He}^{4}(1 \%)$ \\
\hline half-field & $20 / 1$ & 6. & 2.5 & 14.5 & 11.8 & 5.1 & 42,40 & $\pm(27,50)$ & $\mathrm{He}^{4}, \mathrm{H}$ \\
\hline
\end{tabular}

similar to the conventional hydrogen in deuterium scheme, extensively studied in the present and past fusion devices. Figure (1.b) shows the power repartition predicted by the ICRF codes for $\mathrm{He}^{3}, \mathrm{~T}$, and electrons. The fraction absorbed by deuterium is at most a few percents, and the fraction to $\mathrm{He}^{4}$ is completely negligible. The predicted power repartition has the same trends for all the codes, although they differ in magnitude. However, the agreement in the trends point to the fact that traces of $\mathrm{He}^{3}$ increase the rf power directly absorbed by ions, and only about $3 \%$ of $\mathrm{He}^{3}$ is enough to reach the maximum of power directly absorbed by $\mathrm{He}^{3}$. In view of injecting $\mathrm{He}^{3}$ in ITER to improve the ICRF performances, this is a favourable prediction, since $\mathrm{He}^{3}$ is particularly rare and thus expensive. Around the concentration of optimal $\mathrm{He}^{3}$ absorption, namely 3\%, figure (1.b) shows the deposition profiles of the dominant absorbing species. CYRANO and LION codes predict a higher on-axis absorption than EVE and TORIC.

The agreement in the trends of the power repartition is good also in the case of half-field ITER phase, as shown in Figure (2.b). The optimum of $\mathrm{H}$ absorption is around $5 \%$ of the $\mathrm{H}$ concentration, and direct electron heating becomes quickly dominant as the $\mathrm{H}$ concentration is increased. The agreement of the absorbed power profiles between EVE and TORIC is much better than in the full-field ITER phase, as shown in figure (2.c). At high H concentration, where mode conversion starts to play a role and thus electron absorption predicted by EVE/TORIC increases, the discrepancy between LION and EVE/TORIC appears smaller in both the global and the profiles of the absorbed power. 

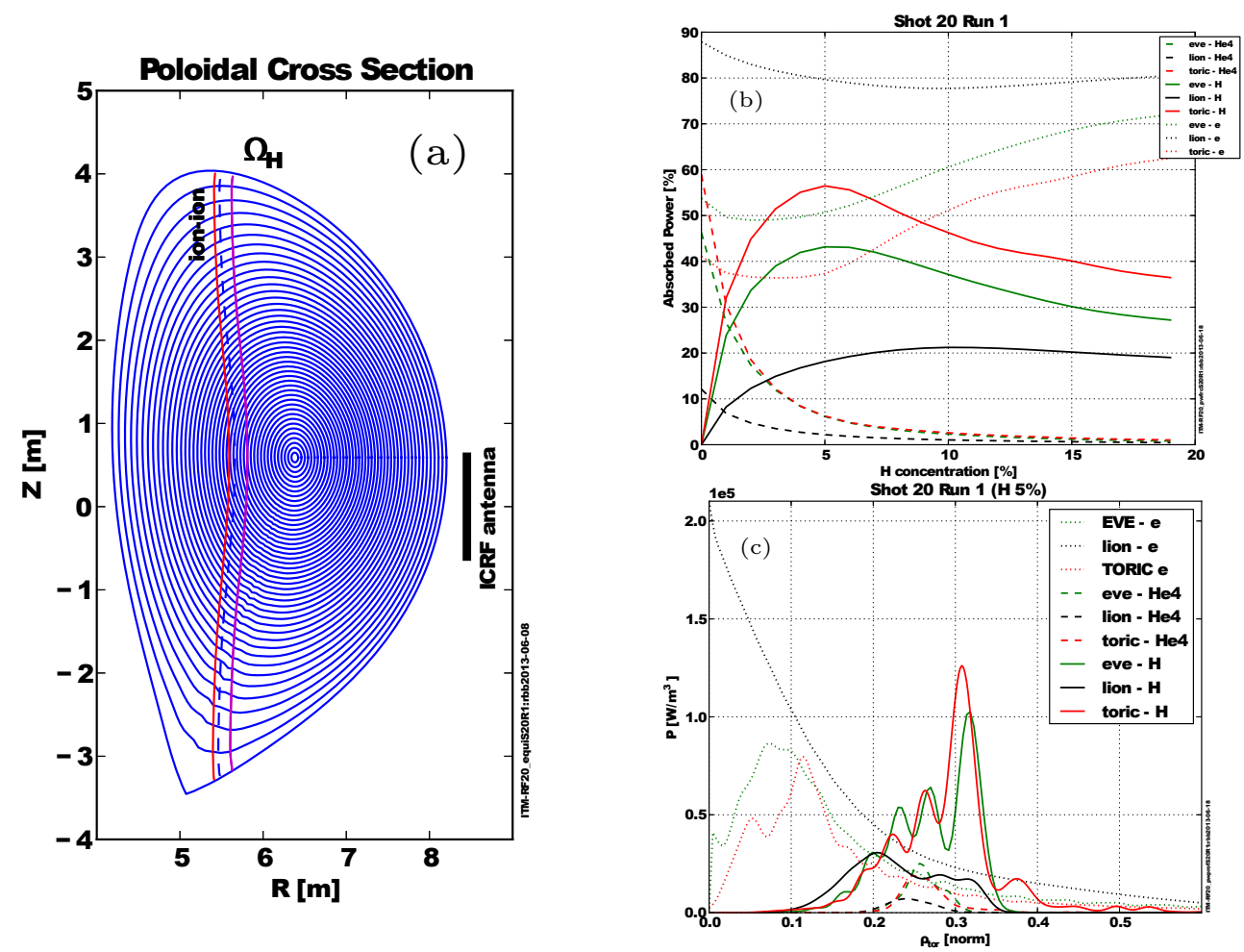

FIGURE 2. Half-field ITER case: (a) Poloidal cross section of the magnetic configuration; (b) Power repartition as function of the hydrogen concentration; (3) Absorbed-power profiles for $5 \%$ of hydrogen. In these simulations $\mathrm{f}=42 \mathrm{MHz}$ and $n_{\varphi}=27$.

\section{CONCLUSIONS}

This initial phase of the benchmark activity pointed out both agreements and discrepancies among the ICRF codes imported on the ITM platform. A detailed investigation of the differences will require an analysis both of the models used in each code and of the implemented numerical methods. In particular, the numerical resolution and boundary conditions have to be carefully addressed. In the near future, some thinking of how to model the currents flowing in the antenna straps will be done, together with a better translation of the geometry of the ITER antenna in the antennas CPO. More fundamental is the undertaken construction of modules for the ionic and electronic quasilinear operator, necessary to interface ICRF codes with FP solvers. This is in view of performing selfconsistent ICRF simulations to provide sources to transport and turbulence codes.

Acknowledgments This work, supported by the European Communities under the contract of Association between EURATOM-IPP, CEA, KTH, LPP-ERM-KMS, EPFL, and CNR, was carried out within the framework of the Task Force on Integrated Tokamak Modelling of the European Fusion Development Agreement. The views and opinions expressed herein do not necessarily reflect those of the European Commission.

\section{REFERENCES}

1. A. Becoulet et al. Comp. Phys. Comm., 177(1,2): 55 - 59, 2007.

2. F. Imbeaux,et al. Comp. Phys. Comm., 181(6):987 - 998, 2010.

3. G. Manduchi, et al.. Fusion Engineering and Design, 83:462 - 466, 2008.

4. O. Sauter and S.Yu. Medvedev. Comp. Phys. Comm., 184(2):293 - 302, 2013.

5. R.V. Budny, et al. Nuclear Fusion, 52(2):023023, 2012.

6. R. Dumont, Nuclear Fusion 49, 075033 (2009).

7. P. U. Lamalle, PhD thesis - Université de Mons (1994) LPP-ERM/KMS Laboratory Report 101.

8. L. Villard, S. Brunner, and J. Vaclavik, Nuclear Fusion 35, 1173 (1995).

9. M. Brambilla, and R. Bilato, Nuclear Fusion 49, 085004 (2009).

10. H. Lütjens, A. Bondeson, and O. Sauter, Computer Physics Communications 97, 219 - 260 (1996). 